D) Check for updates

Cite this: Polym. Chem., 2021, 12, 4934

Received 28th June 2021 Accepted 20th July 2021

DOI: $10.1039 / \mathrm{d} 1$ py00872b

rsc.li/polymers

\title{
Influence of the tetraalkoxysilane crosslinker on the properties of polysiloxane-based elastomers prepared by the Lewis acid-catalysed Piers-Rubinsztajn reaction $\uparrow$
}

\author{
Andrew M. Hickman, (D) ${ }^{\text {a,b }}$ Nikola Chmel, (D) ${ }^{c}$ Neil R. Cameron, (D) b,d \\ Daniel J. Keddie iD ${ }^{\mathrm{e}}$ and Tara L. Schiller (iD *a,b
}

\begin{abstract}
We investigate the preparation of polysiloxane-based networks under solvent-free, ambient conditions using the Lewis acid catalysed Piers-Rubinsztajn (PR) reaction of hydride-terminated siloxanes with various tetrafunctional alkoxysilanes (tetraethoxysilane, tetrapropoxysilane, tetra- $n$-buxoxysilane, tetra-sbutoxysilane, tetra-s-butoxysilane, and tetrakis(2-ethylbutoxy)silane) as crosslinkers. We explore the effects of polysiloxane chain length and crosslinker alkyl group on the rheological performance of the elastomers. By analysing the reaction progress by grazing angle Fourier-transform infrared spectroscopy (FTIR) and determining the rheological properties of the resulting materials, we show that the use of linear or branched alkoxysilanes strongly influences the morphology and properties of these network polymers. We have shown the PR process can be tailored to reliably produce homogeneous, polysiloxane network materials. This work provides information on the relative rates of network formation under ambient conditions with an emphasis on the impact of crosslinker alkyl chain length. Our results show that electronics and sterics both play critical roles in influencing the rate of the curing reaction. Crucially, we newly demonstrate the benefit of a having tertiary carbon $\alpha$ to the SiO reaction centre, as is the case for the tetra-s-butoxysilane crosslinker, for delivering exceptionally rapid network cure and a concomitant enhancement in storage modulus of the resultant materials.
\end{abstract}

\section{Introduction}

Polysiloxanes (also commonly known as silicones) are polymers that consist of alternating covalently-linked silicon and oxygen atoms, with organic functionality on the silicon atoms. They are employed extensively in a range of applications such as sealants $;^{1}$ contact lenses, ${ }^{2}$ adhesives, ${ }^{3}$ bakeware, ${ }^{4}$ cosmetics ${ }^{5}$ and implants. ${ }^{6}$ This is due to the desirable properties of siloxanebased materials including hydrophobicity, thermal stability, oxygen permeability, biocompatibility, and optical transparency. ${ }^{7}$

Current methods of polysiloxane-based network preparation is comprised of three main techniques: tin-catalysed moisture

\footnotetext{
${ }^{a}$ WMG, University of Warwick, Coventry CV4 7AL, UK.

E-mail: T.L.Schiller@warwick.ac.uk

${ }^{b}$ Department of Materials Science and Engineering, Monash University, 22 Alliance Lane, Clayton 3800, Victoria, Australia

${ }^{c}$ Department of Chemistry, University of Warwick, Coventry CV4 7AL, UK

${ }^{d}$ School of Engineering, University of Warwick, Coventry CV4 7AL, UK

${ }^{e}$ Faculty of Science and Engineering University of Wolverhampton,

Wolverhampton WV1 $1 L Y$, UK

$\dagger$ Electronic supplementary information (ESI) available. See DOI: 10.1039/ d1py00872b
}

cure; high-temperature radical cure; and platinum-catalysed hydrosilylation. ${ }^{8}$ Achieving reproducibility in elastomeric properties is difficult through high-temperature radical cure. ${ }^{7}$ Tinbased catalysed reactions offer greater accuracy for material properties but as there is potential for environmental harm from tin residues, other routes are more desirable., ${ }^{4,9}$ Platinum-catalysed hydrosilylation is an efficient reaction, able to proceed at low catalyst loadings of $\leq 30 \mathrm{ppm}$ and resulting in no by-products. ${ }^{10}$ Platinum-based catalysts are expensive, with Pt accounting for approximately $30 \%$ of the material cost. ${ }^{11}$ The catalyst is retrievable, however the recovery process is difficult and inefficient, with only $10-15 \%$ of Pt catalyst being reusable. ${ }^{12}$

A newer method for making siloxane bonds is the PiersRubinsztajn (PR) reaction. This reaction does not require transition metal catalysts and importantly proceeds efficiently at room temperature. ${ }^{13}$ Initial use of $\mathrm{PR}$ reaction for siloxane bond formation was displayed as polysiloxane production by coupling dihydridosilanes with dialkoxysilanes through a polycondensation reaction. ${ }^{13}$

The PR reaction proceeds through the complexation of the Lewis acid, $\mathrm{B}\left(\mathrm{C}_{6} \mathrm{~F}_{5}\right)_{3}$, with a $\mathrm{Si}-\mathrm{H}$ intermediate $\mathbf{A}$ (see Scheme 1). ${ }^{14,15}$ Addition of an alkoxysilane to this intermedi- 
ate leads to the reversible formation of the hydridosilane oxonium complex B (Scheme 1). From intermediate $\mathbf{B}$ there are three potential pathways: (i) dissociation back to starting reagents (black pathway, Scheme 1); (ii) reductive elimination of the alkyl group, resulting in the desired siloxane formation and alkane by-product (blue pathway, Scheme 1); and (iii) undesired metathesis of the complex, transferring of the hydride to produce a new hydridosilane and alkoxysilane (red pathway, Scheme 1). Experimental evidence shows negligible metathesis, with siloxane formation the predominant outcome (pathway (ii) Scheme 1). ${ }^{16,17}$

In the context of siloxane-based polymeric materials, the metal-free Lewis acid PR reaction has demonstrated utility for siloxane network formation. ${ }^{18-20}$ This reaction leads to a gaseous alkane by-product, which influences network formation and can lead to fragile foams. ${ }^{21}$ With this is mind, we sought to develop methods for reproducible gels, without foaming, under atmospheric conditions with a view to a wider scope of application for these materials.

In this paper we consider the impact of crosslinker alkyl chain length on rates of solvent-free gel network formation. The resultant materials, characterised by rheometry and FTIR, provide insights on the factors which influence the properties of the resultant networks.

\section{Experimental}

\section{Materials and methods}

$\alpha, \omega$-Hydride-terminated poly(dimethylsiloxane) (PDMS) 400-500 $\mathrm{g} \mathrm{mol}^{-1}\left(M_{\mathrm{n}} \sim 800 \mathrm{~g} \mathrm{~mol}^{-1}, 1 \mathrm{a}\right.$, calculated by endgroup analysis from ${ }^{1} \mathrm{H}$ NMR spectroscopy), $\alpha, \omega$-hydride-terminated poly(dimethylsiloxane) (PDMS) $1000-1100 \mathrm{~g} \mathrm{~mol}^{-1}\left(M_{\mathrm{n}}\right.$ $\sim 1170 \mathrm{~g} \mathrm{~mol}^{-1}, \mathbf{1 b}$, calculated by end-group analysis from ${ }^{1} \mathrm{H}$ NMR spectroscopy), tetra- $n$-butoxysilane 4 , tetra- $s$-butoxysilane 6, tetrakis(2-ethylbutoxy)silane $\mathbf{5}$ were purchased from Gelest (Germany). Tris(pentafluorophenyl)borane $\left(\mathrm{B}\left(\mathrm{C}_{6} \mathrm{~F}_{5}\right)_{3}\right.$, tetrapropoxysilane $3, n$-hexane, $n$-heptane, toluene and ethanol were purchased from Sigma-Aldrich (UK). Tetraethoxysilane 2 was purchased from Fischer Scientific (UK). All were used as received.

\section{Preparation of $\mathrm{B}\left(\mathrm{C}_{6} \mathrm{~F}_{5}\right)_{3}$ catalyst solutions}

A $0.1 \mathrm{M}$ stock solution was prepared by adding $\mathrm{B}\left(\mathrm{C}_{6} \mathrm{~F}_{5}\right)_{3}$ $(0.512 \mathrm{~g})$ into a volumetric flask $(10 \mathrm{~mL})$ and filling up to the mark with toluene. A subsequent $0.01 \mathrm{M}$ stock solution was

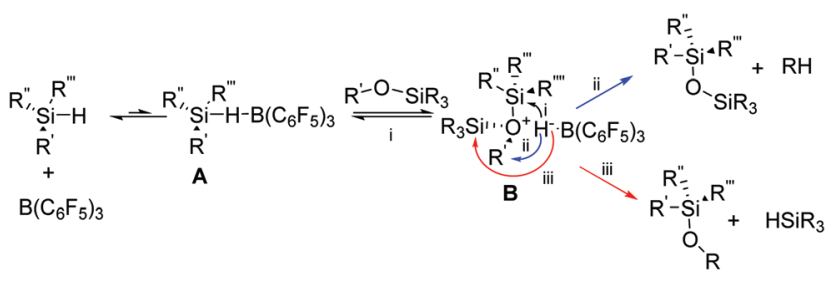

Scheme 1 The reaction pathways for Piers-Rubinsztajn reaction. ${ }^{17}$ made by adding an aliquot $(1 \mathrm{~mL})$ from the $0.1 \mathrm{M}$ stock solution to a volumetric flask $(10 \mathrm{~mL})$ and filled up to the mark with toluene.

\section{General preparation of silicone elastomer}

For silicone elastomer preparation, $\alpha, \omega$-hydride terminated PDMS (1a or 1b) was mixed with tetraalkoxysilane crosslinker $(2,3,4,5$, or 6$)$ at a ratio of $2.125: 1$ (e.g. 1a $=1.7 \mathrm{mmol}$, $0.85 \mathrm{~mL}: 4=0.8 \mathrm{mmol}, 0.285 \mathrm{~mL}$ ) respectively, using a vortex mixer for $1 \mathrm{~min}$. The catalyst $\left(\mathrm{B}\left(\mathrm{C}_{6} \mathrm{~F}_{5}\right)_{3}\right)$ is added $(20 \mu \mathrm{L}, 0.01$ $\mathrm{M}, 2 \times 10^{-4} \mathrm{mmol}, 0.024 \mathrm{~mol} \%$ to PDMS) to the silicone and crosslinker solution and immediately mixed on a vortex mixer for 10-20 seconds. The reaction solution was then left to cure at room temperature for 5-30 minutes. The curing was monitored and determined by visual state change from liquid to solid and gentle probing of sample.

\section{Characterisation}

Fourier transform infrared spectroscopy (FTIR). Infrared spectra of pre- and post-cured samples were obtained using a Jasco FTIR 4200 spectrometer, equipped with VeeMAX II with attenuated total reflectance (ATR), Pike Technologies accessory (grazing angle $30^{\circ}$; ZnSe crystal). Measurements were collected at a resolution of $2 \mathrm{~cm}^{-1}$ with 128 scans per sample. Background spectra was collected to remove atmospheric $\mathrm{CO}_{2}$ and $\mathrm{H}_{2} \mathrm{O}$ peaks from sample measurements. Spectra were collected while continuous purging the instrument with $\mathrm{N}_{2}$ to minimise fluctuations in peaks for $\mathrm{CO}_{2}$ and atmospheric $\mathrm{H}_{2} \mathrm{O}$. Spectra were analysed using Origin 2019b.

Rheology. Rheological measurements were taken on an Anton Paar MC501 rheometer (Graz, Austria) using a parallel plate with a diameter of $8 \mathrm{~mm}$ and a measuring gap of $0.18 \mathrm{~mm}$. The change of phase from solution to gel was monitored by measuring the storage moduli and loss moduli. The force was kept constant at $0 \mathrm{~N}$, with the temperature of the Peltier plates was kept at $25{ }^{\circ} \mathrm{C}$ during all measurements. Stock solutions for each composition, including initiator, were prepared and stored on ice to eliminate early onset of reaction. $25 \mu \mathrm{L}$ aliquot of a stock solution was used for each measurement. The reaction was measured with constant frequency of $6.28 \mathrm{rad} \mathrm{s}^{-1}$ and constant strain of $10 \%$. The amplitude sweep was performed at a constant frequency of $6.28 \mathrm{rad} \mathrm{s}^{-1}$ was used and the strain was increased logarithmically from $1-500 \%$. The frequency sweep was conducted at a constant strain of $10 \%$ and the frequency was increased logarithmically from 1-550 $\mathrm{rad} \mathrm{s}^{-1}$. All data was analysed using the rheocompass (Anton Paar, Austria) software.

Nuclear magnetic resonance (NMR) spectroscopy. ${ }^{1} \mathrm{H}$ NMR spectra were collected for hydride-terminated polydimethylsiloxanes 1a and 1b, using a Bruker Avance $400 \mathrm{MHz}$, at room temperature in deuterated chloroform $\left(\mathrm{CDCl}_{3}\right)$. The number average molar mass $\left(M_{\mathrm{n}}\right)$ was calculated using the integration of $\mathrm{Si}-\underline{\mathrm{H}}$ peak at $\delta 4.7 \mathrm{ppm}$ and $\mathrm{Si}^{-\mathrm{CH}_{3}}$ peaks between $\delta$ $-0.1-0.25 \mathrm{ppm}$. The residual solvent peak ( $\delta 7.26 \mathrm{ppm})$ was used as solvent. ${ }^{22}$ 


\section{Results and discussion}

\section{Optimisation of network formation}

Siloxane preparation catalysed by metal-free based compounds is a particularly attractive process, due to the cost and environmental issues metal-based catalysts can pose. Our initial examination of network formation involved experiments using a low molar mass hydride-terminated PDMS 1a, tetraethoxysilane 2 as crosslinker and $n$-hexane as solvent; foamed networks were formed (see Experimental section for stoichiometry, Table S1, $\uparrow$ entry 1). Following the success of the PR reaction 1a with 2, we investigated the effect of the alkyl group of the tetraalkoxysilane crosslinker using the ethyl 2, propyl 3, n-butyl 4, 2-ethylbutyl 5, and $s$-butyl 6 analogues for Piers-Rubinsztajn network formation (see Scheme 2). We observed that an increase in alkyl length of the crosslinker generally led to networks which were denser in appearance (see Fig. S1 $\dagger$ ). Preparation of materials with the bulkier 2-ethylbutyl 5 proved unsuccessful; samples only partially cured with the materials remaining liquid. It is known that larger alkoxysilane ' $R$ ' groups result in a decrease in reaction rate due to the increase in sterics. ${ }^{21}$

Following our initial successes, we then sought to prepare siloxane elastomers, without foaming, via the PR reaction. here we investigated the impact of the tetraalkoxysilane crosslinker alkyl chain length on rate of gelation as well as the resultant materials properties. To avoid the foaming seen in the previous samples, we ascertained that the rate of reaction needed to be low enough to allow the bulk of gas evolved from the reaction to escape during network formation, but remain sufficiently high to allow for complete cure. In this context, we first sought to reduce the rate of reaction by lowering the $\mathrm{B}\left(\mathrm{C}_{6} \mathrm{~F}_{5}\right)_{3}$ catalyst concentration, in relation to conditions reported in previous studies. ${ }^{21,23}$ These experiments (see Table $\mathrm{S} 1 \dagger)$ showed that, as expected, the $\mathrm{B}\left(\mathrm{C}_{6} \mathrm{~F}_{5}\right)_{3}$ catalyst concentration greatly influences the rate of reaction (including gaseous alkane production). The initial reactions discussed above were undertaken using a relatively high catalyst amount $\left(2 \times 10^{-3} \mathrm{mmol} ; 20 \mu \mathrm{L}\right.$ of $0.1 \mathrm{M}$ stock solution). These resulted samples that were brittle and highly-disordered, open celled foams (see Fig. S1(a)†). Brook et al. ${ }^{21}$ have reported comparable outcomes when studying the same mechanism. In their study an increase in crosslinker alkyl chain length, from methyl to propyl, resulted in reduced foaming with the materials having a more closed cell, disordered appearance, as evidenced by SEM. ${ }^{21}$

Upon decreasing the amount of $\mathrm{B}\left(\mathrm{C}_{6} \mathrm{~F}_{5}\right)_{3}$ catalyst $\left(5 \times 10^{-4}\right.$ mmol; $50 \mu \mathrm{L}$ of $0.01 \mathrm{M}$ stock solution) we observed a reduced the rate of cure, with the reactions producing either partially foamed samples or gels with voids (see Fig. 1(a)). Further reduction of catalyst using aliquots $\left(2 \times 10^{-4} \mathrm{mmol} ; 20 \mu \mathrm{L}\right.$ of $0.01 \mathrm{M}$ stock solution) proved effective at reducing voids, enabling production of defect-free elastomers (see Fig. 1(b)). Thus, this was the amount of catalyst used for all subsequent elastomer preparations. (a)

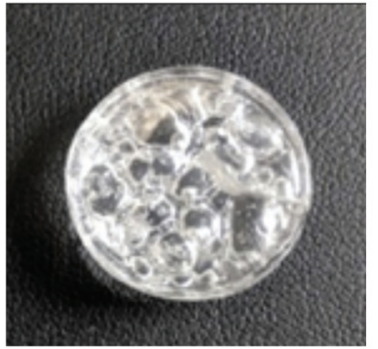

(b)

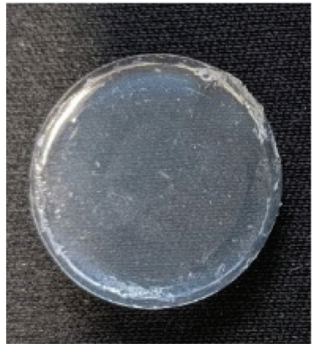

Fig. 1 Digital photographs of bulk samples of PDMS-based polymer networks illustrating the further reduction of foaming upon optimisation of the reaction conditions. Samples were prepared by reacting hydrideterminated PDMS 1 a with tetra- $n$-butoxysilane 4 and: (a) $5 \times 10^{-4} \mathrm{mmol}$ $\mathrm{B}\left(\mathrm{C}_{6} \mathrm{~F}_{5}\right)_{3}$ and (b) $2 \times 10^{-4} \mathrm{mmol} \mathrm{B}\left(\mathrm{C}_{6} \mathrm{~F}_{5}\right)_{3}$.

(a)

(b)

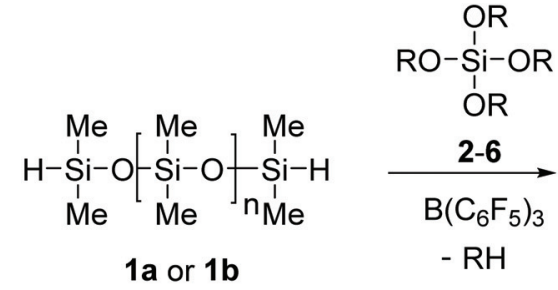

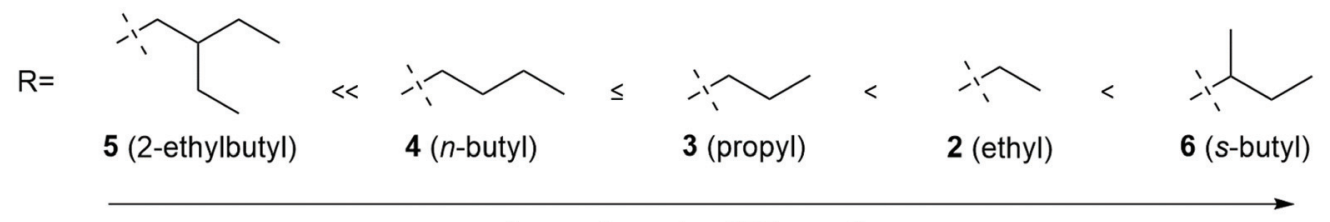

Increasing rate of $\mathrm{PR}$ reaction

Scheme 2 (a) Polysiloxane-network formation via the Piers-Rubinsztajn reaction using hydride terminated siloxanes (1a, 1b) and tetraalkoxysilanes $(2-6)$, and (b) the alkyl ' $R$ ' groups of the tetraalkoxysilanes (2-6) displayed in order of increasing relative rate of reaction. Note the formation of the alkane by-product $\mathrm{RH}$. 
In all of the reactions discussed above, $n$-hexane was used as a solvent; it also acts as a heat sink for the exothermic PR reaction. However, due to its volatility, $n$-hexane can affect the outcome of the polysiloxane material produced by acting as a blowing agent, ${ }^{21}$ increasing the likelihood of foamed products. This was observed for our samples.

Upon changing the solvent from $n$-hexane to $n$-heptane we observed a reduction in visual gas evolution during the PR curing. This led to a decrease in voids present within the elastomers (not shown). Note, the boiling point of $n$-heptane is $\sim 30-40{ }^{\circ} \mathrm{C}$ greater than $n$-hexane, ${ }^{24}$ and the difference in the latent heats of vaporisation of approximately $10 \mathrm{~kJ} \mathrm{~mol}^{-1}$; $n$-heptane reduces the volume of additional gas produced during the PR curing under otherwise the same reaction conditions.

The cured samples prepared using either $n$-hexane or $n$-heptane were found to contract following solvent evaporation which led to sample fracture (possibly due to increased strain present within the network in a contracted state). Omission of solvent from the reaction mixtures was investigated in an attempt to overcome this problem. Without solvent, a reduction in swelling and a decreased incidence of sample fracture was observed. While the occurrence of voids was not completely eliminated, solventless reactions were deemed the best approach for preparing our samples; all samples discussed in subsequent sections were prepared without solvent.

\section{Investigating rate of network formation}

To examine the effect of changing the alkyl R-groups of the tetraalkoxysilane crosslinker (i.e., ethyl 2, propyl 3, n-butyl 4, 2-ethylbutyl 5, and $s$-butyl 6) on the rate and extent of PR network formation and the final mechanical properties these possess, the curing reactions were studied by both grazingangle FTIR and rheology. Note, these systems display induction periods of varying lengths of time prior to cure, consistent with that previously reported for the PR reaction. ${ }^{25}$ The commercial samples we used were not pre-dried and therefore contain unknown, varying amounts of moisture. This results in these differing induction periods. ${ }^{26}$

Pre- and post-cure grazing-angle FTIR for the samples prepared from PDMS 1a was first undertaken. Absorbance peaks at $2126 \mathrm{~cm}^{-1}$ and $907 \mathrm{~cm}^{-1}$, were not found in the post-cure measurements (see Fig. 2, top), indicating consumption of Si$\mathrm{H}^{27,28}$ groups. Complete cure was estimated for systems using 1a with ethyl 2, propyl 3, $n$-butyl 4 or $s$-butyl 6 (see entries 1-3 $\& 5$, Table 1), achieving almost full consumption of $\mathrm{Si}-\mathrm{H}$ functional groups PDMS 1a with ethyl 2 (see entry 1, Table 1; Fig. 3(a)) reacted rapidly, indicated by sharp increase in modulus at $\sim 30$ seconds, this was followed by a gradual increase to a plateau modulus of $82 \mathrm{kPa} .+$

\$This differing modulus plateau may be due to forceful gas evolution of ethyl 2 reducing contact with the plates. The shear from the rheology plates promotes mixing and once the gas escapes the system, better contact can be established with the solid material, leading to a gradual increase in storage modulus.

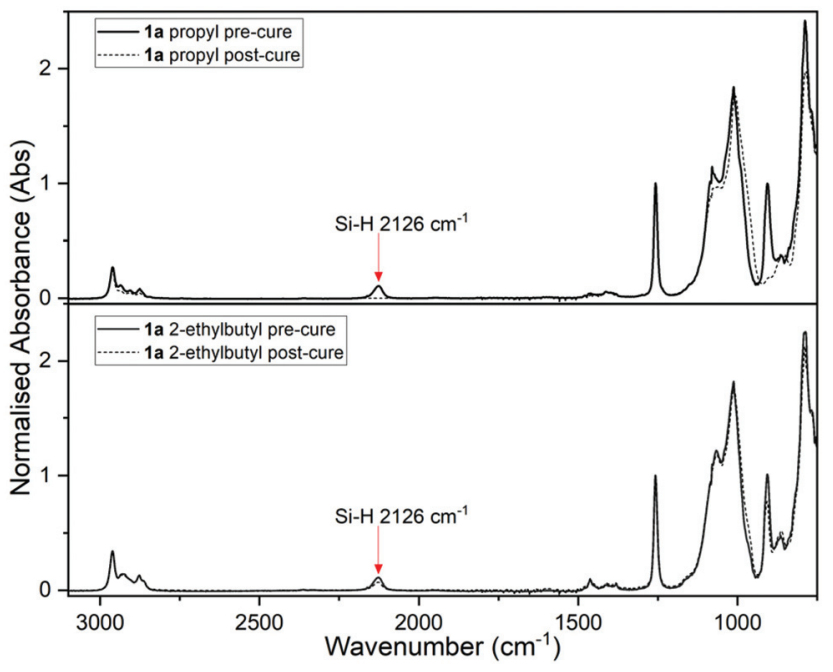

Fig. 2 Absorbance normalised $30^{\circ}$ grazing angle FTIR spectra for (top) PDMS system 1a propyl 3 pre- and post-cure measurements, $\mathrm{Si}-\mathrm{H}$ peak at $2126 \mathrm{~cm}^{-1}$ disappears from pre- to post-cure measurements, and (bottom) PDMS system 1a 2-ethylbutyl 5 pre- and post-cure measurements, $\mathrm{Si}-\mathrm{H}$ peak found at $2126 \mathrm{~cm}^{-1}$ decreases from pre- to post-cure measurements.

Table 1 Results from FTIR and rheology measurements of elastomer systems

\begin{tabular}{|c|c|c|c|c|}
\hline Entry & $\begin{array}{l}\text { Hydride- } \\
\text { terminated } \\
\text { PDMS }\end{array}$ & Tetraalkoxysilane & $\begin{array}{l}\text { Conversion }{ }^{c} \\
(\%)\end{array}$ & $\begin{array}{l}G^{\prime d} \\
(\mathrm{kPa})\end{array}$ \\
\hline $1^{a}$ & $1 \mathbf{a}$ & 2 (ethyl) & 100 & 82.0 \\
\hline $2^{a}$ & 1a & 3 (propyl) & 100 & 38.4 \\
\hline $3^{a}$ & $1 a$ & 4 (n-butyl) & 100 & 32.6 \\
\hline $4^{a}$ & $1 a$ & 5 (2-ethylbutyl) & 38 & $-^{e}$ \\
\hline $5^{a}$ & $1 a$ & $6(s$-butyl $)$ & 99 & 137 \\
\hline $6^{b}$ & 1b & 2 (ethyl) & 98 & 444 \\
\hline $7^{b}$ & 1b & 3 (propyl) & 94 & 241 \\
\hline $8^{b}$ & 1b & 4 (n-butyl) & 100 & 337 \\
\hline $9^{b}$ & $\mathbf{1 b}$ & 5 (2-ethylbutyl) & 38 & $-^{e}$ \\
\hline $10^{b}$ & 1b & $6(s$-butyl $)$ & 98 & 428 \\
\hline
\end{tabular}

${ }^{a}$ PDMS 1a $M_{\mathrm{n}}=800 \mathrm{~g} \mathrm{~mol}{ }^{-1},[1 \mathrm{a}]=1.7 \mathrm{mmol}$, [alkoxysilane] = $0.8 \mathrm{mmol},\left[\mathrm{B}\left(\mathrm{C}_{6} \mathrm{~F}_{5}\right)_{3}\right]=2 \times 10^{-4} \mathrm{mmol} .{ }^{b}$ PDMS 1b $M_{\mathrm{n}}=1170 \mathrm{~g} \mathrm{~mol}^{-1}$, $[\mathbf{1 b}]=0.85 \mathrm{mmol}$, [alkoxysilane] $=0.4 \mathrm{mmol},\left[\mathrm{B}\left(\mathrm{C}_{6} \mathrm{~F}_{5}\right)_{3}\right]=1 \times 10^{-4}$ mmol. ${ }^{c}$ Conversion \% based on consumption of $\mathrm{Si}-\mathrm{H}\left(2126 \mathrm{~cm}^{-1}\right)$ calculated by $\%=100-((($ post-cure absorbance $) /($ pre-cure absorbance $)) \times$ 100). ${ }^{d}$ Peak storage modulus obtained from rheology analysis. ${ }^{e}$ Not measured due to insufficient cure.

In contrast to the ethyl 2 sample (see entry 1, Table 1; Fig. 3(a)), the propyl 3 and $n$-butyl 4 gelation curves displayed similar characteristics with a peak in $G^{\prime}$ followed by a slight decrease to a plateau of $37.2 \mathrm{kPa}$ and $32.1 \mathrm{kPa}$ respectively (see entries $2 \& 3$, Table 1; Fig. 3(b \& c)). This suggests these are more homogeneous than the ethyl sample, lacking voids.t This is also consistent with the qualitative evidence discussed above (for images see Fig. $\mathrm{S} 1 \dagger$ ).

The curing of the PDMS 1a/s-butyl 6 sample also displayed sharp increase in modulus (to $137.2 \mathrm{kPa}$ ); resulting, after a small decrease, in the highest modulus observed (129.6 kPa) 
(a)

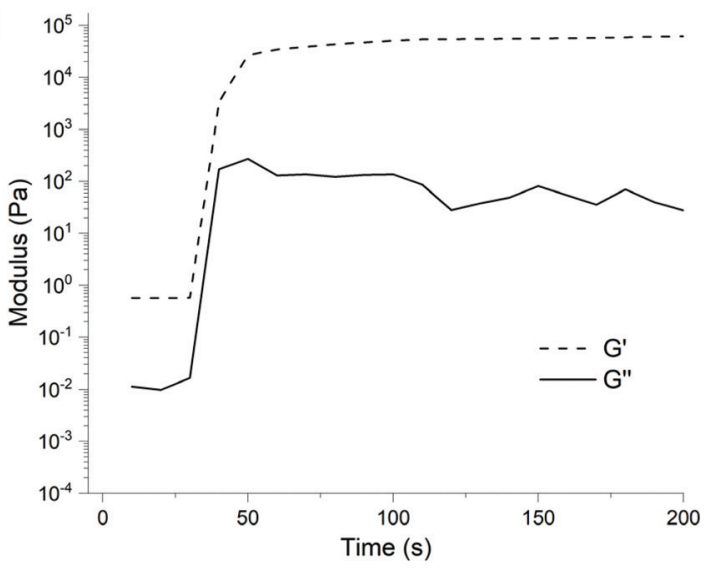

(c)

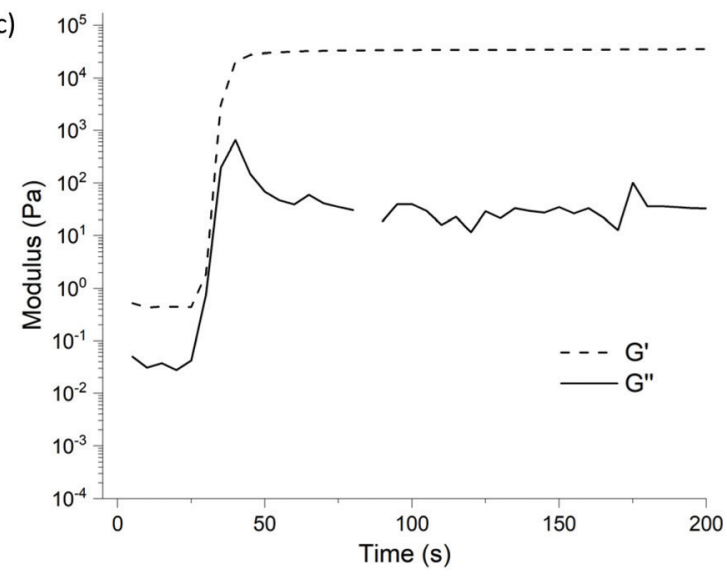

(b)

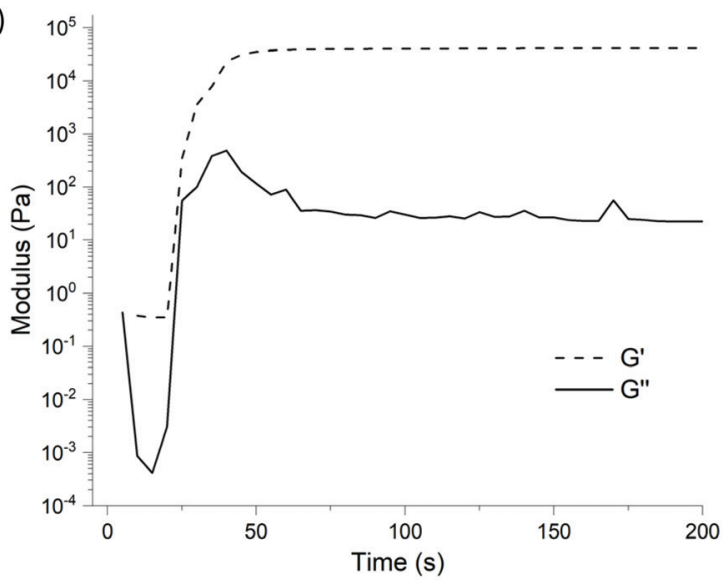

(d)

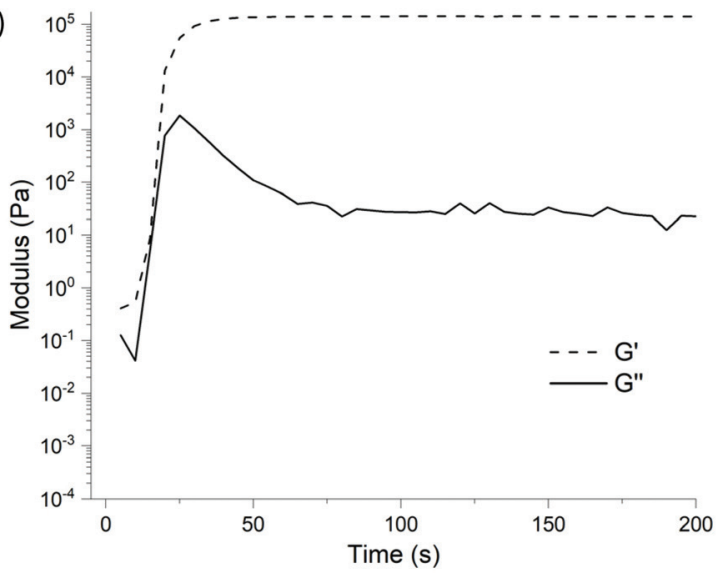

Fig. 3 Time-resolved rheology gelation curves over 200 seconds shown by G' and G" for elastomers derived from PDMS 1a and the (a) ethyl 2 note differing transition to plateau by gradual increase in modulus, (b) propyl 3, (c) n-butyl 4, and (d) s-butyl 6 alkoxysilane (also see entries 1-5, Table 1). Note the omitted data as per Table 1.

(see Fig. 3(d) and entry 5, Table 1). It was observed that the reaction rate was higher, with the reaction able to proceed at $0{ }^{\circ} \mathrm{C}$; in contrast this temperature retarded the reactions using the crosslinkers $\mathbf{2}-\mathbf{5}$.

The clear difference between $s$-butyl 6 crosslinker and the other crosslinkers $\mathbf{2 - 5}$ is the presence of a tertiary carbon $\alpha$ to the SiO group; the other crosslinkers 2-5 all have secondary carbons in this position (see Scheme 2). Since the predominant reaction pathway (see Scheme 1, (ii)) proceeds via a hydride shift to the alkoxy carbon atom, we postulate that this difference in electronics between 6 and the other crosslinkers (i.e. 2-5) at this reaction centre is key. An enhanced ability for the tertiary carbon(s) on 6 to stabilise a (partial) positive charge, which is expected to contribute to the transition state, would account for the observed increase in reaction rate. Indeed, this crude hypothesis is consistent with a detailed mechanistic summary of the $\mathrm{PR}$ reaction published by Chojnowski et al. ${ }^{25}$

Increasing the bulk of the crosslinker alkyl chain (with minimal change to electronics of the carbon $\alpha$ to the SiOgroup)using the 2-ethylbutyl 5 (see entry 4, Table 1) resulted in significantly lower \% cure in the final 'cured' sample; it remained liquid, indicating only partial cure. The FTIR analysis supports this, displaying only partial consumption of $\mathrm{Si}$ $\mathrm{H}$ (see Fig. 2, bottom), with the calculated percentage of conversion being 38\%.§ A gelation curve was not obtained as 2-ethylbutyl 5 did not cure on the rheometer, this is consistent with the FTIR data. $s$-butyl 6 exhibited an efficient reaction; resulting in an increased final $G^{\prime}$ and a high conversion percentage. This was determined to be the optimal crosslinker (see entry 5 , Table 1 ; Fig. $3(\mathrm{~d})$ ).

§Conventional gelation theory states that an $\mathrm{A}_{2} \mathrm{~B}_{4}$ system (where $\mathrm{A}$ is an $\alpha, \omega$-hydride terminated PDMS and B is a tetraalkoxysilane) three-dimensional structure forms beyond $33 \%$ conversion. ${ }^{31,32}$ entry 4 , Table 1 has an estimated conversion of $38 \%$; although beyond the theoretical 33\% required for network formation, there is a margin of error in the calculation. In addition, FTIR only analyses a small region of the sample, both of which will contribute to variations in conversion data. The decrease in estimated percentage conversion, compared to other formulations, is likely due to the increase in steric hindrance by the larger, branched alkyl chain, leading to a reduction in rate of the PR reaction. 


\section{Networks prepared with longer chain length PDMS}

Tailoring thermo-mechanical properties is vital to the application of polymeric materials. In this context, chain length between crosslinks is known to influence these properties. ${ }^{29,30}$ The rate of cure is also influenced by the chain length of endgroup functional polymers.

In this context, the reaction conditions optimised for lower molar mass PDMS 1a were adjusted for a higher molar mass hydride-terminate PDMS $1 \mathbf{b}\left(M_{\mathrm{n}} 1170 \mathrm{~g} \mathrm{~mol}^{-1}\right)$, to ensure reaction volume and stoichiometries were comparable. For these reactions we maintained a similar volume $(\sim 1 \mathrm{~mL})$ and as such the amount of polymer, crosslinker and catalyst were halved (i.e., $0.85 \mathrm{mmol}$ polymer, $0.4 \mathrm{mmol}$ crosslinker and $1 \times$ $10^{-4} \mathrm{mmol}$ catalyst). Keeping the reaction volume similar allowed for more direct comparison between the samples derived from 1a and 1b polymer groups; samples of larger volume (by using the same number of moles of $\mathbf{1 b}$ as $\mathbf{1 a}$ ) led to more defects in the resultant elastomers, due to the greater distance the gaseous alkane by-product needed to travel to escape the network.

The PDMS 1b derived samples prepared using the ethyl 2, propyl 3, or $n$-butyl 4 crosslinkers readily cured, yielding con- versions of $98 \%, 93 \%$ and $99 \%$, respectively (see Table 1, entries 6,7 , and 8 respectively). These three samples post-cure were solid materials, with no tackiness from unreacted starting reagents. The PDMS 1b/2-ethylbutyl 5 sample did not completely cure, with the sample again remaining in a liquid state (see entry 9, Table 1). From FTIR analysis, the conversion was calculated to be $38 \%$, comparable to the PDMS 1a/2-ethylbutyl 5 sample (see entry 4, Table 1), which also exhibited partial curing.

The ethyl 2 sample displayed the highest initial $\mathrm{G}^{\prime}$, with a steep increase to $444 \mathrm{kPa}$, followed by a gradual decrease to $388 \mathrm{kPa}$ (see Fig. 4(a) and entry 6, Table 1). Changing the crosslinkers alkyl chain to propyl 3 resulted in a decrease in the initial gelation storage modulus, peaking at $241 \mathrm{kPa}$. The initial peak in $\mathrm{G}^{\prime}$ of the propyl 3 system (see Fig. 4(b) and entry 7, Table 1) was followed by a decrease to $151 \mathrm{kPa}$, similar to observations for the ethyl 2 system. The storage modulus ( $\left.\mathrm{G}^{\prime}\right)$ of the $n$-butyl 4 sample (peaked at $337 \mathrm{kPa}$ see Fig. 4(c) and entry 8 , Table 1), however the transition from the initial steep period to peak $\mathrm{G}^{\prime}$ was more gradual, indicating a reduction in reaction rate in comparison to ethyl 2 and propyl 3 systems. After the modulus peaked, a gradual decrease was seen again, reaching $224 \mathrm{kPa}$. The $s$-butyl 6 sample initial curing led to a (a)

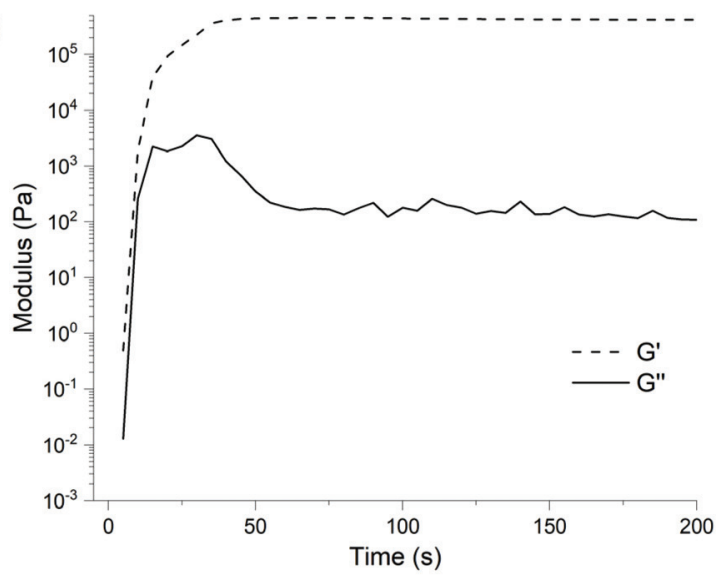

(c)

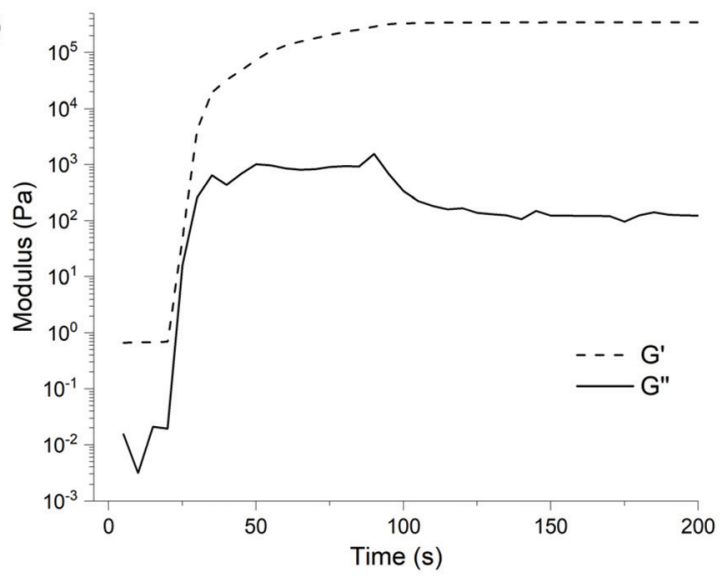

(b)

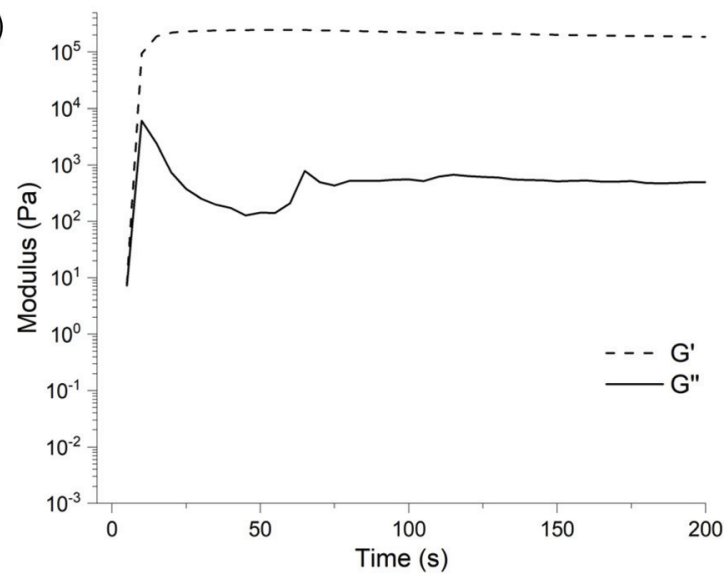

(d)

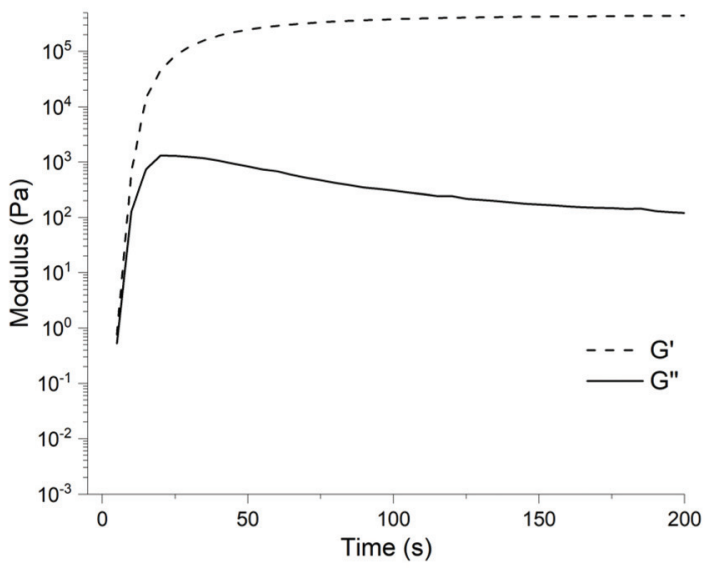

Fig. 4 Time-resolved rheology gelation curves over 200 seconds shown by $G$ ' and $G$ " for elastomers derived from PDMS $1 b$ and the (a) ethyl 2, (b) propyl 3, (c) n-butyl 4, and (d) s-butyl 6 alkoxysilane (also see entries 6-10, Table 1). Note the omitted data as per Table 1. 
peak $G^{\prime}$ of $428 \mathrm{kPa}$ (see Fig. 4(d) and entry 10, Table 1), the best from all crosslinkers tested. Interestingly, the gelation curve did not exhibit the same gradual decrease in storage modulus as seen in samples using PDMS $\mathbf{1 b}$ and the ethyl 2, propyl 3 and $n$-butyl 4 (see entries 6 and 7, Table 1). This system (1b/6) does display a higher storage modulus relative to crosslinkers 3 \& $\mathbf{4}$, similar to the analogous system using the smaller PDMS (1a/6). Further investigation is required into $s$-butyl 6 systems to understand why this phenomenon occurs, which is outside the scope of our current work.

\section{Conclusions}

Parameters for reproducible, solvent-free PR systems for the synthesis of polysiloxane (silicone) elastomers were established. A range of alkoxysilane crosslinkers ethyl 2, propyl 3, $n$-butyl 4, 2-ethylbutyl 5 and $s$-butyl 6 were investigated with two siloxane polymers 1a $\left.\left(M_{\mathrm{n}} \sim 800 \mathrm{~g} \mathrm{~mol}\right)^{-1}\right) \& \mathbf{1 b}\left(M_{\mathrm{n}} \sim\right.$ $\left.1170 \mathrm{~g} \mathrm{~mol}^{-1}\right)$. Increasing the crosslinker alkyl chain length from ethyl 2 to $n$-butyl 4 resulted in an observable change in reaction rate and the appearance of the final product. The propyl $\mathbf{3}$ and $n$-butyl $\mathbf{4}$ crosslinkers resulted in decreased foaming compared to ethyl 2, resulting in elastomers. The percentage cure, determined by FTIR, for crosslinkers ethyl 2, propyl 3, $n$-butyl 4 and $s$-butyl $\mathbf{6}$ were estimated to be between 94-100\% for both polymers 1 a and 1b, whilst the branched 2-ethylbutyl 5 resulted in a much lower cure of $38 \%$.

Time-resolved rheological studies showed gelation curves with a rapid increase in modulus, after a short induction period, followed by a plateau for each of the crosslinkers ethyl 2, propyl 3, n-butyl 4 and $s$-butyl 6. The ethyl 2 derived samples displayed a noticeable difference in their gelation curves, exhibiting a more gradual transition to the plateau. Samples containing $s$-butyl 6 displayed a larger increase in modulus comparative to any of the linear counterparts. Use of a larger branched alkyl chain crosslinker (2-ethylbutyl 5) reduced the rate of reaction considerably, leading to incomplete curing in both systems.

This work has provided an insight into the influence of crosslinker alkyl chain on PR silicone elastomer formation. We have shown that increasing the size of the alkyl group away from the $\mathrm{SiO}$ reaction centre, reduces the rate of reaction. In contrast we have demonstrated that altering the electronics of crosslinker, by changing the group directly bonded to the $\mathrm{SiO}$ reaction centre from secondary to tertiary, greatly enhances the reaction rate. In this context, the $s$-Butyl 6 displays both a more rapid cure and a higher storage modulus than the other crosslinkers tested and is the ideal choice for further investigations in PR elastomer preparation.

\section{Author contributions}

$\mathrm{AH}$ : validation, investigation, drafted original manuscript, review and editing of manuscript; NC: FTIR investigation, review of manuscript; NRC: student co-supervision, review and editing of manuscript; DJK: student co-supervision, conceptualization, review and editing of manuscript; TLS: student main supervision, conceptualization, helped draft original manuscript, review and editing of manuscript.

\section{Conflicts of interest}

There are no conflicts to declare.

\section{Acknowledgements}

The authors would like to thank Dr Daniel Lester from the Warwick Polymer Research Technology Platform for his assistance with the Rheometer and Dr Ahmed Eissa for his assistance with the ${ }^{1} \mathrm{H}$ NMR.

\section{Notes and references}

1 M. J. Owen and J. M. Klosowski, Durability of Silicone Sealants, in Adhesives, Sealants, and Coatings for Space and Harsh Environments, ed. L.-H. Lee, Springer US, Boston, MA, 1988, pp. 281-291.

2 C. S. A. Musgrave and F. Fang, Contact Lens Materials: A Materials Science Perspective, Materials, 2019, 12(2), 261.

3 S. B. Lin, New silicone pressure-sensitive adhesive technology, Int. J. Adhes. Adhes., 1994, 14(3), 185-191.

4 H. Fromme, M. Witte, L. Fembacher, L. Gruber, T. Hagl, S. Smolic, D. Fiedler, M. Sysoltseva and W. Schober, Siloxane in baking moulds, emission to indoor air and migration to food during baking with an electric oven, Environ. Int., 2019, 126, 145-152.

5 A. J. O'Lenick and K. A. O'Lenick, Silicone Polymers in Skin Care, MRS Bull., 2007, 32(10), 801-806.

6 B. Arkles, Silicones in Biomedical Applications. 1983, pp. 749-768.

7 W. Noll, Chemistry and technology of silicones, Elsevier, 2012.

8 M. A. Brook, Silicon in Organic Organometallic, and Polymer Chemistry, John Wiley \& Sons, Inc., New York, 2000.

9 R. de Carvalho Oliveira and R. E. Santelli, Occurrence and chemical speciation analysis of organotin compounds in the environment: A review, Talanta, 2010, 82(1), 9-24.

10 Y. Nakajima and S. Shimada, Hydrosilylation reaction of olefins: recent advances and perspectives, RSC Adv., 2015, 5(26), 20603-20616.

11 C. H. Schuster, T. Diao, I. Pappas and P. J. Chirik, BenchStable, Substrate-Activated Cobalt Carboxylate Pre-Catalysts for Alkene Hydrosilylation with Tertiary Silanes, ACS Catal., 2016, 6(4), 2632-2636.

12 E. D. Lykissa, S. V. Kala, J. B. Hurley and R. M. Lebovitz, Release of Low Molecular Weight Silicones and Platinum from Silicone Breast Implants, Anal. Chem., 1997, 69(23), 4912-4916. 
13 S. Rubinsztajn and J. A. Cella, A New Polycondensation Process for the Preparation of Polysiloxane Copolymers, Macromolecules, 2005, 38(4), 1061-1063.

14 D. J. Parks, J. M. Blackwell and W. E. Piers, Studies on the Mechanism of B(C6F5)3-Catalyzed Hydrosilation of Carbonyl Functions, J. Org. Chem., 2000, 65(10), 3090-3098.

15 W. E. Piers, A. J. V. Marwitz and L. G. Mercier, Mechanistic Aspects of Bond Activation with Perfluoroarylboranes, Inorg. Chem., 2011, 50(24), 12252-12262.

16 D. B. Thompson and M. A. Brook, Rapid assembly of complex 3D siloxane architectures, J. Am. Chem. Soc., 2008, 130(1), 32-33.

17 J. B. Grande, D. B. Thompson, F. Gonzaga and M. A. Brook, Testing the functional tolerance of the Piers-Rubinsztajn reaction: a new strategy for functional silicones, Chem. Commun., 2010, 46(27), 4988-4990.

18 D. J. Keddie, J. B. Grande, F. Gonzaga, M. A. Brook and T. R. Dargaville, Amphiphilic Silicone Architectures via Anaerobic Thiol-Ene Chemistry, Org. Lett., 2011, 13(22), 6006-6009.

19 J. Zhang, Y. Chen, P. Sewell and M. A. Brook, Utilization of softwood lignin as both crosslinker and reinforcing agent in silicone elastomers, Green Chem., 2015, 17(3), 18111819.

20 M. A. Brook, New Control Over Silicone Synthesis using SiH Chemistry: The Piers-Rubinsztajn Reaction, Chem. Eur. J., 2018, 24(34), 8458-8469.

21 J. B. Grande, A. S. Fawcett, A. J. McLaughlin, F. Gonzaga, T. P. Bender and M. A. Brook, Anhydrous formation of foamed silicone elastomers using the Piers-Rubinsztajn reaction, Polymer, 2012, 53(15), 3135-3142.

22 H. E. Gottlieb, V. Kotlyar and A. Nudelman, NMR Chemical Shifts of Common Laboratory Solvents as Trace Impurities, J. Org. Chem., 1997, 62(21), 7512-7515.

23 A. S. Fawcett, J. B. Grande and M. A. Brook, Rapid, metalfree room temperature vulcanization produces silicone elastomers, J. Polym. Sci., Part A: Polym. Chem., 2013, 51(3), 644-652.

$24 \mathrm{H}$. Kehiaian, Enthalpies of Vaporization of Organic Compounds; A Critical Review and Data Compilation; Chemical Data Series No. 32, Blackwell Scientific Publications, Oxford, 1985.

25 J. Chojnowski, S. Rubinsztajn, J. A. Cella, W. Fortuniak, M. Cypryk, J. Kurjata and K. Kaźmierski, Mechanism of the B(C6F5)3-Catalyzed Reaction of Silyl Hydrides with Alkoxysilanes. Kinetic and Spectroscopic Studies, Organometallics, 2005, 24(25), 6077-6084.

26 A. F. Schneider, Y. Chen and M. A. Brook, Trace water affects tris(pentafluorophenyl)borane catalytic activity in the Piers-Rubinsztajn reaction, Dalton Trans., 2019, 48(36), 13599-13606.

27 P. Launer and B. Arkles, Infrared Analysis of Organosilicon Compounds, 2013, pp. 175-178.

28 N. Risangud, Z. Li, A. Anastasaki, P. Wilson, K. Kempe and D. M. Haddleton, Hydrosilylation as an efficient tool for polymer synthesis and modification with methacrylates, RSC Adv., 2015, 5(8), 5879-5885.

29 A. H. Clark, M. J. Gidley, R. K. Richardson and S. B. RossMurphy, Rheological studies of aqueous amylose gels: the effect of chain length and concentration on gel modulus, Macromolecules, 1989, 22(1), 346-351.

$30 \mathrm{~J}$. E. Mark, Improved elastomers through control of network chain-length distributions, Rubber Chem. Technol., 1999, 72(3), 465.

31 D. R. Miller and C. W. Macosko, A New Derivation of Post Gel Properties of Network Polymers, Macromolecules, 1976, 9(2), 206-211.

32 W. D. Cook, T. L. Schiller, F. Chen, C. Moorhoff, S. H. Thang, C. N. Bowman and T. F. Scott, Effect of CrossLink Density on Photoplasticity of Epoxide Networks Containing Allylic Dithioether Moieties, Macromolecules, 2012, 45(24), 9734-9741. 